\title{
The impact of parental risk factors on the risk of stroke in type 1 diabetes
}

\author{
Anni Ylinen ${ }^{1,2,3}$. Stefanie Hägg-Holmberg ${ }^{1,2,3} \cdot$ Marika I. Eriksson $^{1,2,3} \cdot$ Carol Forsblom $^{1,2,3} \cdot$ Valma Harjutsalo $^{1,2,3,4}$. \\ Jukka Putaala $^{5} \cdot$ Per-Henrik Groop ${ }^{1,2,3,6}$ [D . Lena M. Thorn ${ }^{1,2,3,7}$. on behalf of the FinnDiane Study Group
}

Received: 18 January 2021 / Accepted: 23 February 2021 / Published online: 15 March 2021

(c) The Author(s) 2021

\begin{abstract}
Background Individuals with type 1 diabetes have a markedly increased risk of stroke. In the general population, genetic predisposition has been linked to increased risk of stroke, but this has not been assessed in type 1 diabetes. Our aim was, therefore, to study how parental risk factors affect the risk of stroke in individuals with type 1 diabetes.

Methods This study represents an observational follow-up of 4011 individuals from the Finnish Diabetic Nephropathy Study, mean age at baseline $37.6 \pm 11.9$ years. All strokes during follow-up were verified from medical records or death certificates. The strokes were classified as either ischemic or hemorrhagic. All individuals filled out questionnaires concerning their parents' medical history of hypertension, diabetes, stroke, and/or myocardial infarction.

Results During a median follow-up of 12.4 (10.9-14.2) years, 188 individuals (4.6\%) were diagnosed with their first ever stroke; 134 were ischemic and 54 hemorrhagic. In Cox regression analysis, a history of maternal stroke increased the risk of hemorrhagic stroke, hazard ratio 2.86 (95\% confidence interval 1.27-6.44, $p=0.011$ ) after adjustment for sex, age, BMI, retinal photocoagulation, and diabetic kidney disease. There was, however, no association between maternal stroke and ischemic stroke. No other associations between parental risk factors and ischemic or hemorrhagic stroke were observed.

Conclusion A history of maternal stroke increases the risk of hemorrhagic stroke in individuals with type 1 diabetes. Other parental risk factors seem to have limited impact on the risk of stroke.
\end{abstract}

Keywords Parental risk factors $\cdot$ Cardiovascular complications $\cdot$ Stroke $\cdot$ Hemorrhagic stroke $\cdot$ Ischemic stroke $\cdot$ Type 1 diabetes

Managed by Massimo Porta.

Per-Henrik Groop

per-henrik.groop@helsinki.fi

1 Folkhälsan Institute of Genetics, Folkhälsan Research Center, Biomedicum Helsinki, Helsinki, Finland

2 Department of Nephrology, University of Helsinki and Helsinki University Hospital, Helsinki, Finland

3 Research Program for Clinical and Molecular Metabolism, Faculty of Medicine, University of Helsinki, Helsinki, Finland

4 National Institute for Health and Welfare, Helsinki, Finland

5 Helsinki University Hospital and University of Helsinki, Neurology, Helsinki, Finland

6 Department of Diabetes, Central Clinical School, Monash University, Melbourne, VIC, Australia

7 Department of General Practice and Primary Health Care, University of Helsinki and Helsinki University Hospital, Helsinki, Finland

\section{Abbreviations \\ CI Confidence interval \\ eGFR Estimated glomerular filtration rate \\ FinnDiane The Finnish Diabetic Nephropathy Study}

\section{Background}

Diabetes is a significant risk factor for stroke, especially in young people [1]. The incidence of stroke in individuals with type 1 diabetes is $310-475$ per 100,000 person-years [2-4], which is about fivefold higher than in the general population [5]. Stroke is a cerebrovascular disease commonly divided into two subgroups, ischemic and hemorrhagic. Hemorrhagic stroke is less common than ischemic stroke but associated with worse prognosis and higher mortality rate [6]. Risk factors for stroke, such as age, hypertension, and smoking are similar in the general population $[7,8]$ as in type 2 [9] and type 1 diabetes [10]. One of the traditional 
risk factors for stroke, atrial fibrillation, does not relate to increased risk of stroke in type 1 diabetes, at least in younger individuals [10]. In type 1 diabetes, however, also diabetesrelated factors increase the risk of stroke, and the risk factor profiles for ischemic and hemorrhagic stroke differ [10]. Additional risk factors for ischemic stroke include diabetic kidney disease, poor glycemic control, and insulin resistance, while for hemorrhagic stroke the presence of diabetic kidney disease and diabetic retinopathy, and lower BMI increases the risk $[10,11]$.

In addition to modifiable risk factors, genetic risk factors are also important for the development of stroke. A large genome-wide association study identified different loci for ischemic and hemorrhagic stroke. [12] To evaluate the genetic risk in routine clinical practice, a simple tool is to assess the family history and parental risk factors. Studies on parental risk factors show parental stroke to be associated with increased risk of stroke in their offspring [13, 14]. It has also been shown that hypertension and stroke occur concurrently in families [15]. In type 1 diabetes the impact of parental risk factors on the risk of stroke has not been studied. Our aim was, therefore, to explore a potential familial predisposition to stroke and specifically how hypertension, diabetes, stroke, and/or myocardial infarction in the parents of individuals with type 1 diabetes affect their risk of stroke.

\section{Methods}

All individuals are part of the Finnish Diabetic Nephropathy (FinnDiane) Study, which is a multicenter study founded in 1997, including 77 study centers (Additional file 1). The study aims to uncover genetic and environmental risk factors for micro- and macro-vascular complications in type 1 diabetes. This study is an observational follow-up study, previously described in detail [16]. Of the 4,467 individuals with type 1 diabetes that have participated in the FinnDiane study before 1.1.2013, we excluded 350 with incomplete parental history, 96 with stroke prior to baseline, and 10 with traumatic or uncertain stroke, resulting in 4011 eligible individuals for the study. Type 1 diabetes was defined as age at onset below 40 years and initiation of insulin therapy within one year from diagnosis.

The study protocol has been approved by the Ethics Committee of the Helsinki and Uusimaa Hospital District. Each individual gave their written informed consent prior to their inclusion in the study. The study was conducted in accordance with the Declaration of Helsinki.

Data were collected at regular visits to the attending physician. Information regarding medical history and medication was registered. Anthropometrics were measured at the visit (body weight, height, and blood pressure). Blood samples were drawn and analyzed for $\mathrm{HbA}_{1 \mathrm{c}}$, lipids and lipoproteins, as well as serum creatinine. The glomerular filtration rate (eGFR) was estimated with the Chronic Kidney Disease Epidemiology Collaboration (CKD-EPI) equation [17]. Diabetic kidney disease was defined as history of severely increased albuminuria in two out of three urine collections ( $\geq 200 \mu \mathrm{g} / \mathrm{min}$ or $\geq 300 \mathrm{mg} / 24 \mathrm{~h}$ ), history of renal transplantation, or being on dialysis. Cardiovascular disease was defined as history of myocardial infarction, coronary revascularization, and/or lower limb amputation or revascularization. History of smoking was defined as previous or current smoking. The participating individuals filled out questionnaires concerning their medical history, smoking habits, and family history. In this study, we used the information available from the questionnaires regarding the medical history of the parents. The questionnaires on parental history included information on year of birth and death, history of antihypertensive treatment, myocardial infarction, stroke, and diabetes. In case a parent had diabetes, the age at diagnosis $(<30,30-60$, or $>60$ years) and type of treatment (diet, oral hypoglycemic agents, or insulin) was documented. Based on this the condition could be defined as type 1 diabetes (age at onset $<30$ years and insulin treatment), type 2 diabetes (age at onset $>60$ years or treatment with oral hypoglycemic agents or diet), or unclassifiable diabetes (age between 30 and 60 at onset or no information on age at onset or type of medication).

Follow-up data. Individuals who suffered a stroke were identified from death certificates retrieved from Statistics Finland and from the Finnish Care Register for Health Care based on the ICD-10 (codes I60-I64). Medical records, computed tomography, and magnetic resonance images were then ordered for these identified individuals from the hospital where they had been treated. All strokes were verified by a stroke neurologist (J.P.) and classified as either ischemic or hemorrhagic according to a protocol previously described in detail $[2,11]$. Follow-up data were available until 31 December 2012.

Statistical analyses. We tested all continuous variables for normal distribution. For parametric continuous variables, we used $t$ tests to compare means, and for non-parametric ones, we used Mann-Whitney U-tests. The results are presented as mean with standard deviation or as median with interquartile intervals, respectively. We used the $\chi^{2}$-test to test differences in categorical variables between groups.

Cox regression analyses were conducted to assess the independent role of parental risk factors for the first ever stroke during follow-up, as well as for ischemic or hemorrhagic stroke, respectively. Variables were chosen based on the significance in univariable analysis, and the final models included variables independently associated with the outcome. The final model for the Cox regression analysis for any stroke included sex, age, systolic blood pressure, $\mathrm{HbA}_{1 \mathrm{c}}$, retinal photocoagulation, and diabetic kidney disease. The 
Cox regression analysis for ischemic stroke included the same variables as for any stroke, with the addition of smoking, whereas the analyses for hemorrhagic stroke included sex, age, BMI, retinal photocoagulation, and diabetic kidney disease. Each parental risk factor was then added to the model separately. The results are presented as hazard ratio with $95 \%$ confidence interval. $p<0.05$ was considered statistically significant. Statistical analyses were performed with SPSS Statistics 24.0 software (IBM Corporation, Armonk, NY, USA) and R version 4.0.0.

\section{Results}

During a median follow-up of 12.4 (10.9-14.2) years, 188 (4.6\%) of the 4,011 study participants were diagnosed with their first ever stroke; 134 (71.3\%) were ischemic and 54 $(28.7 \%)$ were hemorrhagic. The characteristics of the individuals and their parents at baseline, according to the presence or the absence of stroke during follow-up, are shown in Table 1. Individuals with stroke were older, were more often men, and had a longer diabetes duration. Higher blood pressure, unfavorable cholesterol concentrations, higher $\mathrm{HbA}_{1 c}$, lower eGFR, and a history of diabetic kidney disease, retinal photocoagulation, and cardiovascular disease, as well as smoking were more common among the individuals with stroke.

Since the individuals with stroke were older, also their parents were older at baseline, resulting in a higher prevalence of parental risk factors. The prevalence of stroke, myocardial infarction, hypertension, and diabetes was higher for the mothers of individuals with stroke, especially in the any stroke and ischemic stroke groups. (Table 1) After adjustment for age, only maternal stroke was associated with any stroke $(p=0.040)$ and hemorrhagic stroke $(p=0.010)$. Further, in Cox regression analysis, only maternal stroke was associated with increased risk of hemorrhagic stroke in individuals with type 1 diabetes, hazard ratio 2.86 (95\% CI 1.27-6.44, $p=0.011$ ) (Fig. 1, Table 2).

Of the paternal risk factors, only the prevalence of myocardial infarction was higher for the fathers of individuals with any stroke and ischemic stroke. Noteworthy, at baseline, fewer fathers than mothers were alive, and even fewer of the fathers to those that had suffered a stroke were alive. (Table 1). In Cox regression analyses, no significant associations between parental hypertension, myocardial infarction, stroke, and diabetes and the risk of stroke or stroke subtypes appeared (Table 2).

\section{Discussion}

In this large observational follow-up study of individuals with type 1 diabetes, we showed that maternal stroke is associated with increased risk of stroke, especially hemorrhagic stroke. None of the other parental risk factors studied were associated with increased risk of stroke or the stroke subtypes ischemic or hemorrhagic. Our results indicate that familial predisposition to stroke plays a lesser role in type 1 diabetes and that diabetes-related factors, in contrast, play a larger role.

This is the first study, to our knowledge, to assess parental risk factors for risk of stroke in type 1 diabetes. We showed that maternal stroke increases the risk of hemorrhagic stroke by nearly threefold after adjustment for confounders. Similarly, in people without diabetes, parental stroke is associated with a threefold increased risk of stroke in the offspring [13], and familial predisposition to stroke is stronger for hemorrhagic stroke than for ischemic [18]. For the different subtypes of stroke, the familial predisposition differs, as shown by a large Swedish population-based study, where the type of stroke in siblings had different impact on the stroke subtype in the index case. Hemorrhagic stroke in siblings increased the risk of hemorrhagic stroke, while ischemic stroke in siblings increased the risk of ischemic stroke, but not vice versa. [19] In our study, we did unfortunately not have data on the subtype of stroke in the parents or data on stroke in siblings.

In line with our results, maternal history is more prevalent than paternal history of stroke in individuals with stroke, especially in those with ischemic stroke and in particular women with stroke [20]. In our study, we observed similar findings, but for those with hemorrhagic stroke. It was not possible to assess the parental risk factors separately for men and women with type 1 diabetes, due to lack of statistical power. However, in individuals with type 1 diabetes, we previously observed that men have a higher risk of stroke than women, but this difference is explained by the higher risk of diabetic kidney disease in men [10], and therefore all analyses were adjusted for both sex and diabetic kidney disease in the present study. In contrast to studies in the general population $[15,21]$, we did not observe an association between parental hypertension and risk of stroke. It is possible that the familial predisposition to stroke is of lesser importance in individuals with type 1 diabetes. This risk of stroke is foremost driven by diabetes-related factors, such as diabetic kidney disease, which has been shown to have a strong impact on the risk of stroke in the individuals in whom stroke occurs at a relatively young age [2]. In this case, a less prominent familial predisposition could be masked by the presence of 
Table 1 Characteristics of the participants at baseline according to presence or absence of stroke during follow-up

\begin{tabular}{|c|c|c|c|c|}
\hline & $\begin{array}{l}\text { No stroke } \\
n=3,823\end{array}$ & $\begin{array}{l}\text { Any stroke } \\
n=188\end{array}$ & $\begin{array}{l}\text { Ischemic stroke } \\
n=134\end{array}$ & $\begin{array}{l}\text { Hemorrhagic stroke } \\
n=54\end{array}$ \\
\hline $\operatorname{Men}(\%)$ & 51 & $62 *$ & $62 *$ & 61 \\
\hline Age, years & $37.2 \pm 11.8$ & $45.8 \pm 9.5^{\dagger}$ & $46.7 \pm 9.6^{\dagger}$ & $43.6 \pm 8.9^{\dagger}$ \\
\hline Age at onset, years & $14.0(9.2-22.3)$ & $13.0(7.9-21.7)^{\dagger}$ & $13.2(8.0-23.4)$ & $12.8(7.5-17.1)$ \\
\hline Duration of diabetes, years & $20.4(11.3-30.0)$ & $31.0(24.9-36.8)^{\dagger}$ & $31.8(25.1-37.5)^{\dagger}$ & $29.1(24.3-36.3)^{\dagger}$ \\
\hline BMI, $\mathrm{kg} / \mathrm{m}^{2}$ & $25.0 \pm 3.6$ & $25.0 \pm 3.8$ & $25.4 \pm 3.9$ & $24.0 \pm 3.4$ \\
\hline Systolic blood pressure, $\mathrm{mmHg}$ & $133 \pm 18$ & $148 \pm 23^{\dagger}$ & $150 \pm 23^{\dagger}$ & $145 \pm 23^{\dagger}$ \\
\hline Diastolic blood pressure, $\mathrm{mmHg}$ & $79 \pm 10$ & $82 \pm 11^{\dagger}$ & $82 \pm 11^{*}$ & $83 \pm 12 *$ \\
\hline Total cholesterol, mmol/l & $4.90 \pm 0.96$ & $5.39 \pm 1.10^{\dagger}$ & $5.37 \pm 1.05^{\dagger}$ & $5.41 \pm 1.22 *$ \\
\hline LDL cholesterol, mmol/l & $2.99 \pm 0.87$ & $3.39 \pm 1.00^{\dagger}$ & $3.41 \pm 0.96^{\dagger}$ & $3.34 \pm 1.10^{*}$ \\
\hline HDL cholesterol, mmol/l & $1.34 \pm 0.39$ & $1.29 \pm 0.41$ & $1.27 \pm 0.41 *$ & $1.33 \pm 0.42$ \\
\hline Triglycerides, mmol/1 & $1.01(0.77-1.44)$ & $1.30(0.94-1.92)^{\dagger}$ & $1.27(0.94-1.90)^{\dagger}$ & $1.32(0.96-1.96)^{\dagger}$ \\
\hline $\mathrm{HbA}_{1 \mathrm{c}}, \%(\mathrm{mmol} / \mathrm{mol})$ & $8.37 \pm 1.48(67.9 \pm 16.1)$ & $8.88 \pm 1.29(73.5 \pm 14.1)^{\dagger}$ & $8.88 \pm 1.23(73.5 \pm 13.4)^{\dagger}$ & $8.87 \pm 1.46(73.5 \pm 16.0)^{*}$ \\
\hline $\mathrm{eGFR}, \mathrm{ml} / \mathrm{min} / 1.73 \mathrm{~m}^{2}$ & $100(83-114)$ & $68(38-97)^{\dagger}$ & $70(44-98)^{\dagger}$ & $64(34-97)^{\dagger}$ \\
\hline Diabetic kidney disease, $\%(n)$ & $20.3(722)$ & $60.2(112)^{\dagger}$ & $59.8(79)^{\dagger}$ & $61.1(33)^{\dagger}$ \\
\hline Retinal photocoagulation, \% (n) & $31.4(1,192)$ & $74.7(139)^{\dagger}$ & $75.2(100)^{\dagger}$ & $73.6(39)^{\dagger}$ \\
\hline Cardiovascular disease, $\%(n)$ & $6.6(252)$ & $20.7(39)^{\dagger}$ & $24.6(33)^{\dagger}$ & $11.1(6)$ \\
\hline History of smoking, $\%(n)$ & $45.2(1,702)$ & $61.0(114)^{\dagger}$ & $64.2(86)^{\dagger}$ & $52.8(28)$ \\
\hline \multicolumn{5}{|l|}{ Maternal characteristics } \\
\hline Age, years & $61.0(53.0-70.0)$ & $71.0(65.0-76.0)^{\dagger}$ & $72.0(65.0-76.0)^{\dagger}$ & $69.0(62.8-75.5)^{\dagger}$ \\
\hline Age at death, years & $70.0(59.0-78.0)$ & $73.0(67.0-80.0)^{*}$ & $72.0(64.5-80.8)$ & $76.0(69.0-77.0)$ \\
\hline Mother alive, \% ( $n)$ & $81.6(3,118)$ & $64.4(121)^{\dagger}$ & $62.7(84)^{\dagger}$ & $68.5(37)^{*}$ \\
\hline Hypertension, \% (n) & $33.4(1,162)$ & $42.1(69)^{*}$ & $42.2(49)^{*}$ & $41.7(20)$ \\
\hline Myocardial infarction, $\%(n)$ & $7.5(254)$ & $17.3(28)^{\dagger}$ & $19.0(22)^{\dagger}$ & $13.0(6)$ \\
\hline Stroke, $\%(n)$ & $4.2(142)$ & $11.9(19)^{\dagger}$ & $9.6(11)^{*}$ & $17.4(8)^{\dagger}$ \\
\hline Diabetes, $\%(n)$ & $13.0(461)$ & $19.2(33)^{*}$ & $19.8(24)^{*}$ & $17.6(9)$ \\
\hline \multicolumn{5}{|l|}{ Paternal characteristics } \\
\hline Age, years & $61.0(54.0-69.0)$ & $72.0(61.5-75.0)^{\dagger}$ & $74.0(61.5-76.0)^{\dagger}$ & $70.5(61.0-73.8)^{\dagger}$ \\
\hline Age at death, years & $62.9 \pm 14.2$ & $65.5 \pm 12.6$ & $65.4 \pm 12.4$ & $66.0 \pm 13.6$ \\
\hline Father alive, $\%(n)$ & $65.1(2,453)$ & $38.0(71)^{\dagger}$ & $34.6(46)^{\dagger}$ & $46.3(25)^{*}$ \\
\hline Hypertension, \% (n) & $29.0(921)$ & $32.9(48)$ & $33.7(35)$ & $31.0(13)$ \\
\hline Myocardial infarction, $\%(n)$ & $20.0(644)$ & $29.6(45)^{*}$ & $28.7(31)^{*}$ & $31.8(14)$ \\
\hline Stroke, \% (n) & $5.8(183)$ & $7.8(12)$ & $8.3(9)$ & $6.8(3)$ \\
\hline Diabetes, $\%(n)$ & $14.3(476)$ & $17.7(29)$ & $19.0(22)$ & $14.6(7)$ \\
\hline
\end{tabular}

Data are mean $\pm \mathrm{SD}$, median (interquartile range), or percentage (\%). $p$-values indicate comparison with no stroke. $e G F R$, estimated glomerular filtration rate.

$* p<0.05,{ }^{\dagger} p<0.001$

diabetic kidney disease. In addition, parental hypertension, and maternal hypertension, in particular, has previously been shown to be associated with diabetic kidney disease in type 1 diabetes [22], which might further complicate the interpretation of the data. Due to the low number of stroke events in the individuals without diabetic kidney disease, we were unable to assess the impact of parental risk factors in these individuals separately.
We also assessed the impact of parental history of myocardial infarction and parental diabetes on the risk of stroke and found neither of these to increase the risk of any stroke or stroke subtypes. Parental history of myocardial infarction could indicate a history of atherosclerosis. In type 1 diabetes, the stroke etiology differs from the general population in that a larger proportion of the stroke events are caused by small-vessel disease, whereas large-vessel 


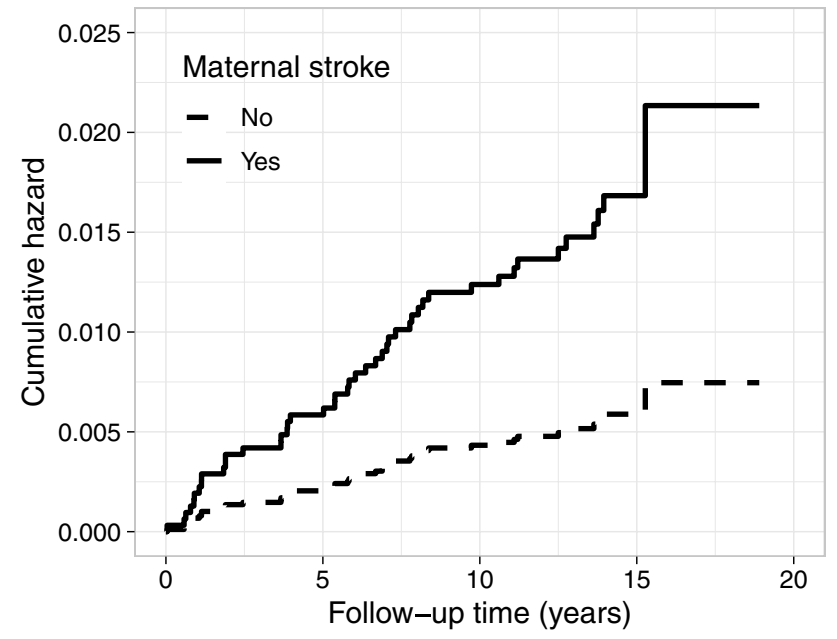

Figure 1 Cumulative hazard for hemorrhagic stroke according to maternal stroke. Maternal stroke associated with hemorrhagic stroke, hazard ratio 2.86 (95\% CI 1.27-6.44), $p=0.011$, adjusted for sex, age, $\mathrm{BMI}$, retinal photocoagulation, and diabetic kidney disease

atherosclerosis is less common [23]. This may explain the lack of association between parental myocardial infarction and risk of stroke in the present study. On the other hand, no such association has been demonstrated for individuals without diabetes either [14].

The strength of this study is the large well-characterized cohort of individuals with type 1 diabetes, with all strokes verified from the medical files by an experienced stroke neurologist. Although the cohort is large, the number of events did not allow extensive sub-analyses, for example, according to sex or absence of diabetic kidney disease. The information about the parents was obtained from the individuals with type 1 diabetes by a questionnaire. Ideally, we would have obtained the information about the parents' medical history by examining them directly, but this was not feasible in the large nationwide FinnDiane cohort. However, the questionnaire enabled us to get information also on those parents that had died. Compared to the mothers, more fathers had died, and they had died at a younger age, which could impact the results regarding the lack of association between paternal risk factors and stroke. Another limitation is that we have only studied the risk factors in the parents, although the medical history of siblings could have an even larger impact on the stroke risk [24].

\section{Conclusions}

In conclusion, we observed that a history of maternal stroke is a significant risk factor for hemorrhagic stroke in individuals with type 1 diabetes. Other parental risk factors seem to have limited impact on the risk, and there is a need for further studies to validate these findings.

Table 2 Associations between parental risk factors and stroke during follow-up

\begin{tabular}{|c|c|c|c|c|c|c|}
\hline & Any stroke $(n=188)$ & $p$-value & Ischemic $\operatorname{stroke}(n=134)$ & $p$-value & Hemorrhagic $\operatorname{stroke}(n=54)$ & $p$-value \\
\hline \multicolumn{7}{|l|}{ Maternal risk factors } \\
\hline Hypertension & $1.01(0.73-1.40)$ & 0.937 & $0.98(0.67-1.44)$ & 0.917 & $1.14(0.63-2.05)$ & 0.671 \\
\hline Myocardial infarction & $1.35(0.87-2.07)$ & 0.179 & $1.46(0.90-2.37)$ & 0.130 & $1.18(0.49-2.83)$ & 0.717 \\
\hline Stroke & $1.49(0.90-2.46)$ & 0.119 & $1.13(0.59-2.15)$ & 0.717 & $2.86(1.27-6.44)$ & 0.011 \\
\hline Diabetes & $1.07(0.73-1.59)$ & 0.720 & $0.96(0.61-1.52)$ & 0.859 & $1.10(0.53-2.30)$ & 0.799 \\
\hline \multicolumn{7}{|l|}{ Paternal risk factors } \\
\hline Hypertension & $1.05(0.73-1.50)$ & 0.810 & $1.13(0.74-1.72)$ & 0.567 & $0.99(0.50-1.95)$ & 0.977 \\
\hline Myocardial infarction & $1.16(0.81-1.67)$ & 0.417 & $1.16(0.76-1.77)$ & 0.501 & $1.44(0.74-2.82)$ & 0.286 \\
\hline Stroke & $0.98(0.54-1.77)$ & 0.938 & $0.99(0.50-1.98)$ & 0.985 & $0.83(0.26-2.72)$ & 0.762 \\
\hline Diabetes & $1.26(0.83-1.89)$ & 0.276 & $1.42(0.89-2.27)$ & 0.143 & $0.93(0.39-2.20)$ & 0.865 \\
\hline \multicolumn{7}{|l|}{ Parental risk factors } \\
\hline Hypertension & $0.97(0.69-1.36)$ & 0.846 & $0.90(0.60-1.35)$ & 0.610 & $1.21(0.64-2.31)$ & 0.560 \\
\hline Myocardial infarction & $1.28(0.91-1.80)$ & 0.160 & $1.32(0.88-1.98)$ & 0.174 & $1.32(0.70-2.51)$ & 0.393 \\
\hline Stroke & $1.23(0.81-1.88)$ & 0.326 & $1.03(0.61-1.73)$ & 0.915 & $1.88(0.92-3.86)$ & 0.085 \\
\hline Diabetes & $1.14(0.82-1.59)$ & 0.431 & $1.22(0.82-1.80)$ & 0.325 & $0.92(0.48-1.75)$ & 0.791 \\
\hline
\end{tabular}

Adjusted Cox regression analyses for association between parental risk factors at baseline and any stroke, ischemic stroke, and hemorrhagic stroke, respectively, during a median of 12.4 years follow-up. Data are hazard ratios with $95 \%$ confidence intervals. For any stroke, the analyses were adjusted for sex, age, systolic blood pressure, $\mathrm{HbA}_{1 \mathrm{c}}$, retinal photocoagulation, and diabetic kidney disease. For ischemic stroke, the analyses were adjusted for the same variables as for any stroke, as well as smoking. For hemorrhagic stroke, the analyses were adjusted for sex, age, $\mathrm{BMI}$, retinal photocoagulation, and diabetic kidney disease. All parental risk factors were analyzed separately 
Supplementary Information The online version contains supplementary material available at (https://doi.org/10.1007/ s00592-021-01694-x).

Acknowledgements We acknowledge all the physicians and nurses at each center participating in the collection of the study population (Additional file 1). We are indebted to Prof. Oili Salonen, Department of Radiology, Helsinki University Hospital, for the help in neuroradiological evaluation.

Authors' contributions P.-H.G. is the guarantor of this work and, as such, had full access to all the data in the study and takes responsibility for the integrity of the data and the accuracy of the data analysis. A.Y. had the main responsibility for analyzing the data and writing the first draft of the paper. S.H.-H., J.P., and L.M.T. contributed to study design, acquisition of data, data analysis, and critical revision of the paper. M.I.E. contributed to data analysis and critical revision of the paper. C.M.F. contributed to study design, acquisition of data, and critical revision of the paper. V.H. contributed to the acquisition of data and critical revision of the paper. P.-H.G. contributed to study design, acquisition of data, critical revision of the paper, and coordination of the study.

Funding Open access funding provided by University of Helsinki including Helsinki University Central Hospital. The study was supported by grants from Folkhälsan Research Foundation, Academy of Finland (275614, 316664), Wilhelm and Else Stockmann Foundation, Liv och Hälsa Society, Novo Nordisk Foundation (NNF OC0013659), Sigrid Jusélius Foundation, Medical Society of Finland, Päivikki and Sakari Sohlberg Foundation, Finnish Foundation for Cardiovascular Research, EVO governmental grants, University of Helsinki, Diabetes Research Foundation, Foundation for the Memory of Dorothea Olivia, Karl Walther and Jarl Perklén, Biomedicum Helsinki Foundation. None of the funding bodies had any role in the study design, collection, analysis, or interpretation of data. Nor had the funding bodies any role in the writing of the report, nor in the decision to submit the paper for publication.

Availability of data and materials The datasets generated and/or analyzed during the current study are not publicly available due to the local legislation and the written consents of the FinnDiane study participants, which do not allow sharing individual-level phenotype data. The data that support the findings are available from the corresponding author upon reasonable request.

\section{Declarations}

Conflict of interests M.I.E. is a shareholder of BCB Medical Oy. P-H.G. has received lecture honoraria from Astellas, AstraZeneca, Boehringer Ingelheim, Eli Lilly, Elo Water, Genzyme, Medscape, MSD, Mundipharma, Novartis, Novo Nordisk, PeerVoice, Sanofi and Sciarc, and he is an advisory board member of AbbVie, Astellas, Astra Zeneca, Bayer, Boehringer Ingelheim, Eli Lilly, Janssen, Medscape, MSD, Mundipharma, Novartis, Novo Nordisk, and Sanofi. The other authors declare no disclosures.

Ethics approval The local ethics committee of each center approved the study protocol, and the study was carried out in accordance with the Declaration of Helsinki.
Informed consent Each participant signed a written informed consent.

Open Access This article is licensed under a Creative Commons Attribution 4.0 International License, which permits use, sharing, adaptation, distribution and reproduction in any medium or format, as long as you give appropriate credit to the original author(s) and the source, provide a link to the Creative Commons licence, and indicate if changes were made. The images or other third party material in this article are included in the article's Creative Commons licence, unless indicated otherwise in a credit line to the material. If material is not included in the article's Creative Commons licence and your intended use is not permitted by statutory regulation or exceeds the permitted use, you will need to obtain permission directly from the copyright holder. To view a copy of this licence, visit http://creativecommons.org/licenses/by/4.0/.

\section{References}

1. Sundquist K, Li X (2006) Type 1 diabetes as a risk factor for stroke in men and women aged 15-49: a nationwide study from Sweden. Diabet Med 23:1261-1267

2. Hagg S, Thorn LM, Putaala J et al (2013) Incidence of stroke according to presence of diabetic nephropathy and severe diabetic retinopathy in patients with type 1 diabetes. Diabet Care 36:4140-4146

3. Secrest AM, Prince CT, Costacou T, Miller RG, Orchard TJ (2013) Predictors of and survival after incident stroke in type 1 diabetes. Diab Vasc Dis Res 10:3-10

4. Janghorbani M, Hu FB, Willett WC et al (2007) Prospective study of type 1 and type 2 diabetes and risk of stroke subtypes: the Nurses' Health Study. Diabet Care 30:1730-1735

5. Harjutsalo V, Thomas MC, Forsblom C, Groop PH, FinnDiane Study Group (2018) Risk of coronary artery disease and stroke according to sex and presence of diabetic nephropathy in type 1 diabetes. Diabet Obes Metab 20:2759-2767

6. Hagg-Holmberg S, Thorn LM, Forsblom CM et al (2017) Prognosis and its predictors after incident stroke in patients with type 1 diabetes. Diabet Care 40:1394-1400

7. Asplund K, Karvanen J, Giampaoli S et al (2009) Relative risks for stroke by age, sex, and population based on follow-up of 18 European populations in the MORGAM Project. Stroke 40:2319-2326

8. Hart RG, Pearce LA, Aguilar MI (2007) Meta-analysis: antithrombotic therapy to prevent stroke in patients who have nonvalvular atrial fibrillation. Ann Intern Med 146:857-867

9. Hankey GJ, Anderson NE, Ting RD et al (2013) Rates and predictors of risk of stroke and its subtypes in diabetes: a prospective observational study. J Neurol Neurosurg Psychiatry $84: 281-287$

10. Hagg S, Thorn LM, Forsblom CM et al (2014) Different risk factor profiles for ischemic and hemorrhagic stroke in type 1 diabetes mellitus. Stroke 45:2558-2562

11. Hagg-Holmberg S, Dahlstrom EH, Forsblom CM et al (2019) The role of blood pressure in risk of ischemic and hemorrhagic stroke in type 1 diabetes. Cardiovasc Diabetol 18(1):1-9

12. Malik R, Chauhan G, Traylor M et al (2018) Multiancestry genome-wide association study of 520,000 subjects identifies 32 loci associated with stroke and stroke subtypes. Nat Genet 50:524 
13. Seshadri S, Beiser A, Pikula A et al (2010) Parental occurrence of stroke and risk of stroke in their children: the Framingham study. Circ 121:1304-1312

14. Jood K, Ladenvall C, Rosengren A, Blomstrand C, Jern C (2005) Family history in ischemic stroke before 70 years of age: the Sahlgrenska Academy Study on Ischemic Stroke. Stroke 36:1383-1387

15. Kondo T, Toyoshima H, Tsuzuki Y et al (2005) Familial aggregation and coaggregation of history of hypertension and stroke. J Hum Hypertens 19:119-125

16. Thorn LM, Forsblom C, Fagerudd J et al (2005) Metabolic syndrome in type 1 diabetes: association with diabetic nephropathy and glycemic control (the FinnDiane study). Diabet Care 28:2019-2024

17. Levey AS, Stevens LA, Schmid CH et al (2009) A new equation to estimate glomerular filtration rate. Ann Intern Med 150:604-612

18. Bruce DG, Van Minnen K, Davis WA et al (2010) Maternal family history of diabetes is associated with a reduced risk of cardiovascular disease in women with type 2 diabetes: the Fremantle Diabetes Study. Diabet Care 33:1477-1483

19. Sundquist K, Li X, Hemminki K (2006) Familial risk of ischemic and hemorrhagic stroke: a large-scale study of the Swedish population. Stroke 37:1668-1673
20. Touze E, Rothwell PM (2008) Sex differences in heritability of ischemic stroke: a systematic review and meta-analysis. Stroke 39:16-23

21. Kadota A, Okamura T, Hozawa A et al (2008) Relationships between family histories of stroke and of hypertension and stroke mortality: NIPPON DATA80, 1980-1999. Hypertens Res 31:1525-1531

22. Thorn LM, Forsblom C, Fagerudd J, Pettersson-Fernholm K, Kilpikari R, Groop PH, FinnDiane Study Group (2007) Clustering of risk factors in parents of patients with type 1 diabetes and nephropathy. Diabet Care 30:1162-1167

23. Putaala J, Liebkind R, Gordin D et al (2011) Diabetes mellitus and ischemic stroke in the young: clinical features and longterm prognosis. Neurol 76:1831-1837

24. Choi JC, Lee JS, Kang SY, Kang JH, Bae JM (2009) Family history and risk for ischemic stroke: sibling history is more strongly correlated with the disease than parental history. J Neurol Sci 284:29-32

Publisher's Note Springer Nature remains neutral with regard to jurisdictional claims in published maps and institutional affiliations. 